\title{
Chapter
}

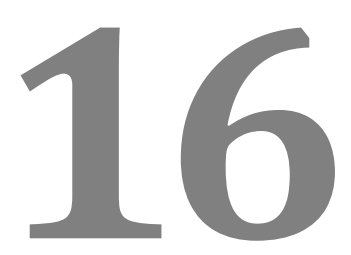

\section{BIOCOMPATIBLE VITAMIN D3 NANOPARTICLES IN DRUG DELIVERY}

\section{Sandeep Palvai and Sudipta Basu*}

Department of Chemistry, Indian Institute of Science Education and Research (IISER)-Pune, Pune, 411008, Maharashtra, India 


\section{Contents}

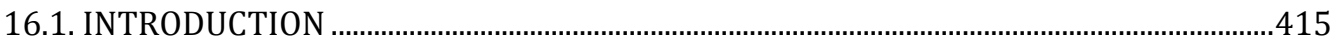

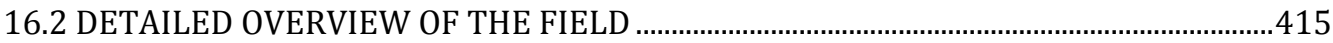

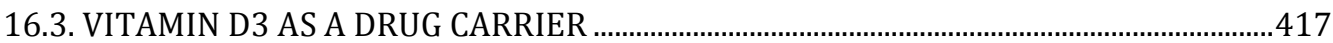

16.3.1. Synthesis and characterization of monodrug loaded vitamin D3

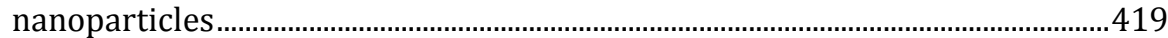

16.3.2. Release of drugs from nanoparticles ................................................................... 421

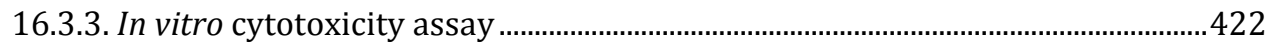

16.3.4. Dual drug loaded vitamin D3 nanoparticles.........................................................424

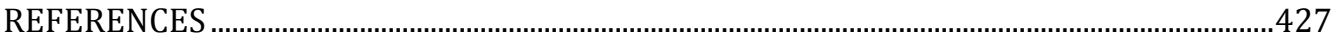




\subsection{INTRODUCTION}

Cancer remains one of the major causes of mortality in the world with 14.1 million new cases and 8.2 million deaths in 2012 [1]. Traditionally, surgical intervention, radiotherapy, chemotherapy, targeted therapy, and immunotherapy have been used extensively to cure cancer [2]. Chemotherapy using small molecule drugs is one of the best strategies in current cancer therapy. However, chemotherapy leads to unwanted bio-distribution into all tissues including the bone marrow, gut, lymphoid tissue, fetus, spermatogenic cells, as well as hair follicles in the body, causing systemic toxicity, and severe side effects in patients [3]. To overcome drug-related toxicities and to guide small molecule chemotherapeutic drugs specifically into tumor tissues, nanotechnology-based tool kits have emerged as an interesting strategy in next-generation cancer therapy.

\subsection{DETAILED OVERVIEW OF THE FIELD}

Nanotechnology is an advanced field of science which involves the design and synthesis of materials in the range of 1-100 nm, offering unprecedented applications in various fields including chemical engineering, biology, physics, food technology, environmental science and many other fields [4]. The advent of nanotechnology has had a great impact on the field of pharmaceutical science, especially in the development of nanomedicine [5]. The emergence of so-called nanomedicine (drug-loaded nanoparticulate systems) offers numerous advantages in treating cancer, including optimized drug loading, improved pharmacokinetic profiles, protection of the payload from premature degradation, controlled and sustained release of drugs, reduced toxicity and efficient in vivo behavior, whereas conventional chemotherapeutics fail to meet these challenges [6]. 


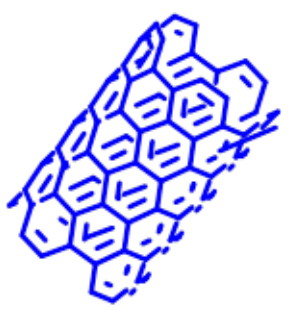

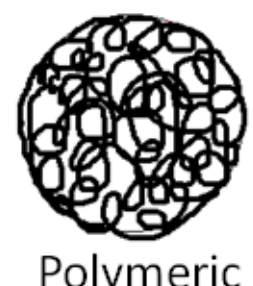

Nanoparticles<smiles>CC(C)C1C(C(C)C)C2C(C(C)C)C(C)CC3C(C)CC(C)C1C32C(C)C</smiles>

Dendrimers

\section{Carbon Nanotubes}

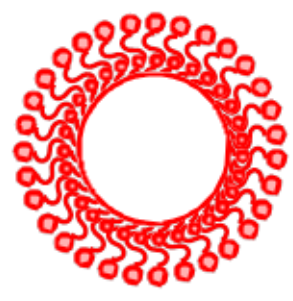

Liposomes

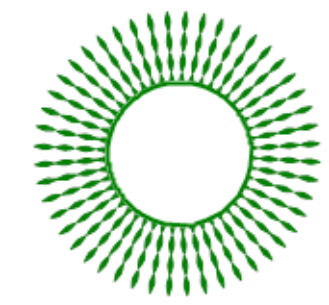

Polymerosomes
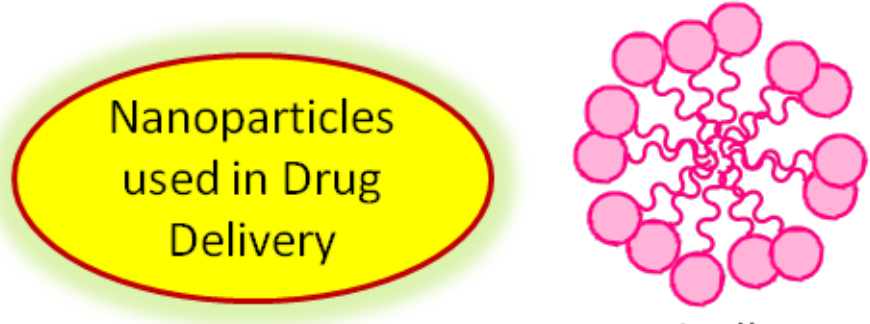

Micelles

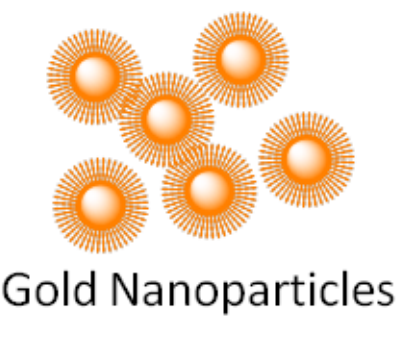

Lipid Coated polymeric

Nanoparticles

Figure 1. Nanoparticles used in drug delivery in cancer

Nanoparticles also have a high surface-area-to-volume ratio which offers surface modification with a variety of different moieties which impart to them certain properties and functionalities such as higher drug loading, prolonged circulation in the blood and further increases in their ability to accumulate in solid tumors via the enhanced permeability and retention (EPR) effect (passive targeting), targeted tissue delivery by attaching specific ligands (active targeting) or by attaching some diagnostic moieties [7]. With the aforementioned properties, nanotechnology is expected to have a dramatic impact on nanomedicine. To date, a large number of organic and inorganic nanoparticles have been developed to encapsulate therapeutic and imaging agents for delivery to the site of action such as liposomes [8,9], dendrimers 
$[10,11]$, polymeric micelles [12,13], polymerosomes [14], solid-lipid nanoparticles [15], gold nanoparticles [16], silicon nanoparticles [17], quantum dots [18] and carbon nanotubes [19] (Figure 1). Although these formulated nanomaterials provide therapeutic advantages over conventional chemotherapy, they also possess unique and complex physicochemical properties that can lead to potential toxicity, resulting in impaired translation of laboratory-based advances into commercial products [20]. Sometimes, the use of unreasonably high quantities of the carrier can lead to problems with carrier toxicity (often resulting from a lack of biodegradability/biocompatibility of vectors used in nanocarrier formulation), metabolism and elimination, or biodegradability [21]. Hence, the evaluation of the biodegradability/biocompatibility of carriers used in nanomedicine is essential for successful translation into the clinic. Very few nanomedicines are currently available on the market for the treatment of cancer. Hence, there is an unmet need to either engineering novel drug delivery systems or to improve existing systems to minimize carrier-related toxicities [22]. This can be achieved by carefully choosing the material composition used in the nanoparticle formulation. Naturally occurring materials are expected to have minimized toxicity and known metabolic clearance mechanism in the body. Hence, it could be anticipated that nanoparticle formulations with naturally occurring materials would be a better approach to overcoming carrier-related toxicities in the successful engineering of nanomedicines. One of the promising naturally occurring candidates for drug delivery purposes is vitamins, which are necessary for our body and are acquired from food sources every day. Recently, we have reported for the first time the synthesis and biological evaluation of biocompatible and biodegradable vitamin D3 nanoparticles for the delivery of various anticancer drugs as monotherapy as well as in combination therapy to target drug resistance in cancer [23].

\subsection{VITAMIN D3 AS A DRUG CARRIER}

Cholecalciferol (vitamin D3) is one of the major compounds of the vitamin D family, produced through ultraviolet irradiation of 7-dehydrocholesterol in the skin [24]. Since vitamin D3 is a naturally occurring, biocompatible, biodegradable and non-toxic molecule, we have chosen this as a vector in the formulation of a novel biocompatible nanoparticle to deliver various clinically approved and extensively used anticancer drugs such as doxorubicin (a DNA damaging agent), PI103 (a phosphatidylinositol-3-kinase inhibitor) and paclitaxel (a microtubule stabilizing agent). 


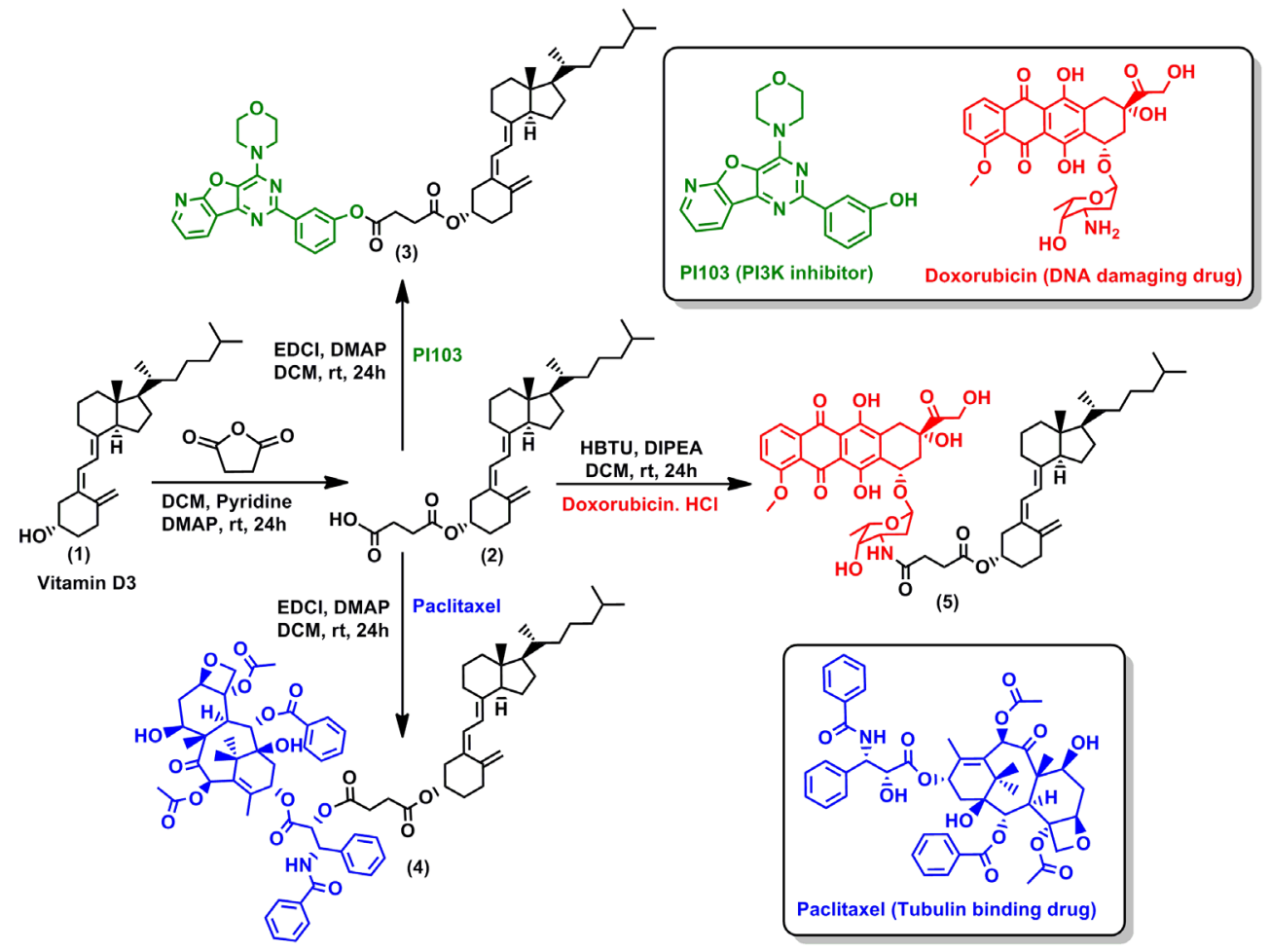

Figure 2. Synthetic scheme of vitamin D3-drug conjugates

The hydroxyl group of vitamin D3 enables the chemical conjugation of drugs. Firstly, vitamin D3 was treated with succinic anhydride in the presence of pyridine and 4-Dimethylaminopyridine (DMAP) to obtain the - $\mathrm{COOH}$ functional group (compound 2 in Figure 2), which further enables the covalent conjugation of drugs through either ester or amide bond formation. PI103 and paclitaxel were conjugated to the $-\mathrm{COOH}$ group by their phenolic and hydroxyl groups, respectively, forming ester bonds by using 1-ethyl-3-(3-dimethylaminopropyl)carbodiimide (EDCI) and DMAP in the presence of dry dichloromethane as solvent to get compounds 3 and 4 (Figure 2). In the same way, doxorubicin was conjugated to the acid group of compound 2 through its secondary amine group by using $N, N, N^{\prime}, N^{\prime}$-Tetramethyl- $O$-(1H-benzotriazol-1-yl)uronium hexafluorophosphate (HBTU) and diisopropylethylamine (DIPEA) in the presence of dry dimethylformamide (DMF) as the solvent to get compound 5 (Figure 2). These drugs conjugated to vitamin D (compounds 3, 4, and 5 in Figure 2) were further used in the synthesis of nanoparticles. 


\subsubsection{Synthesis and characterization of monodrug loaded vitamin D3 nanoparticles}

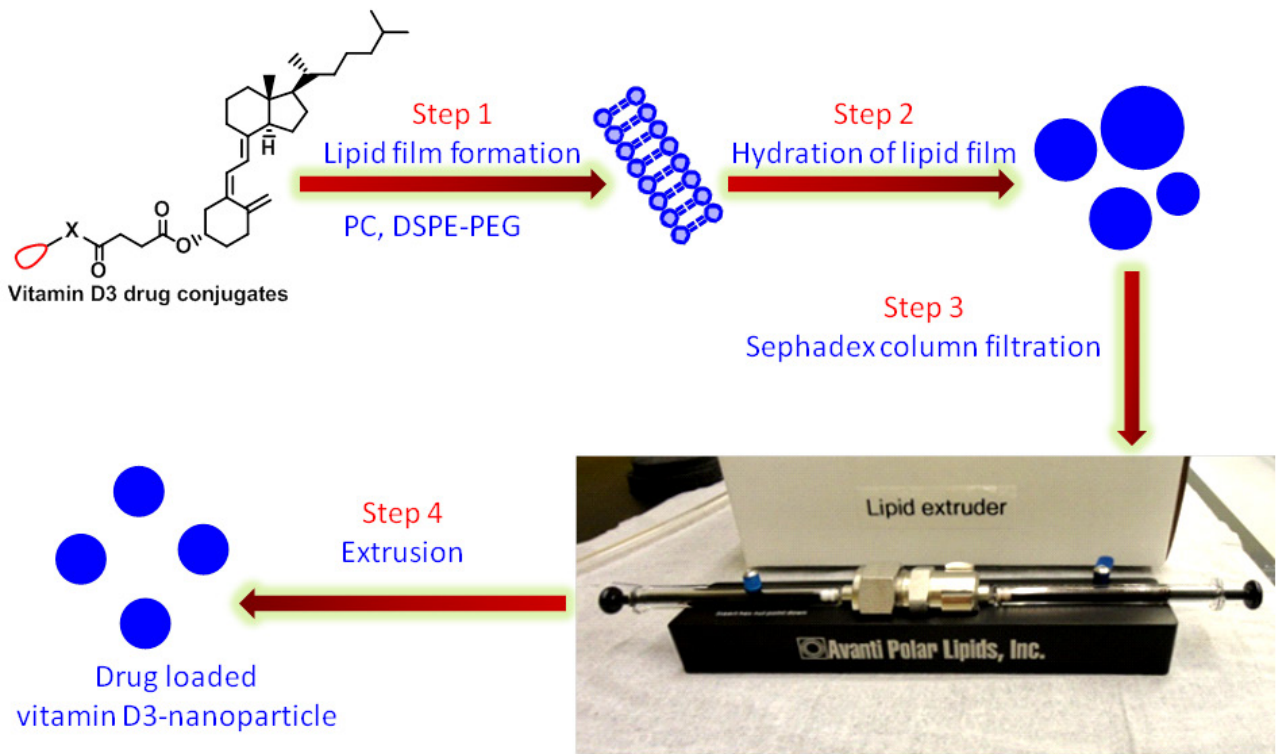

Figure 3. Synthetic scheme for vitamin D3 nanoparticles

Biocompatible vitamin D3 nanoparticles were synthesized from vitamin D3-drug conjugates (compounds 3, 4, and 5) using a solvent evaporation-lipid film hydration-extrusion method (Figure 3). Each drug conjugate 3, 4 or 5 was mixed separately with $\mathrm{L}-\alpha$-phosphatidylcholine (PC), and 1,2-distearoyl-sn-glycero-3-phosphoethanolamine- $N$-[amino(poly(ethylene glycol)) 2000] (DSPE-PEG) in a $1: 2: 0.2$ weight ratio to form a lipid film. This lipid film was hydrated with $1 \mathrm{~mL}$ of $\mathrm{H}_{2} \mathrm{O}$ to get vitamin D3-PI103, vitamin D3-doxorubicin, and vitamin D3-paclitaxel nanoparticles.
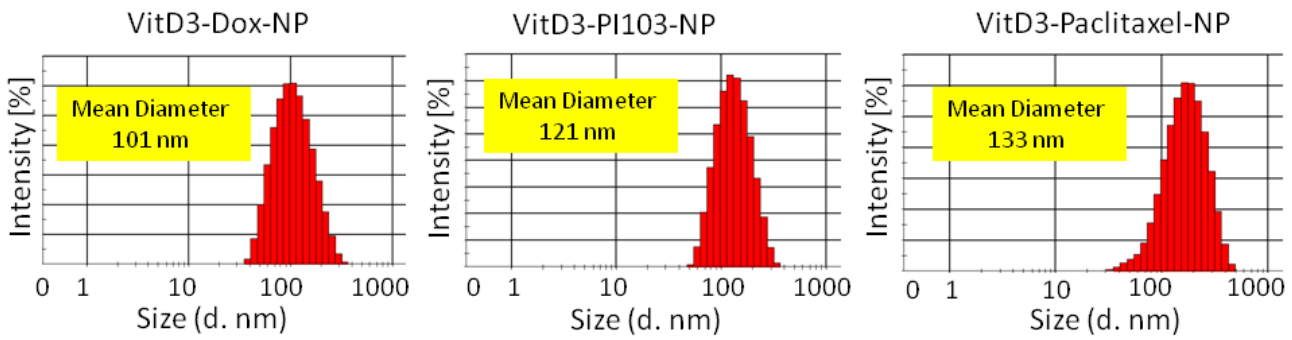

Figure 4. Size distribution of vitamin D3-drug loaded nanoparticles by dynamic light scattering (DLS) 
The size and shape of nanoparticles are very important as they play major roles during blood circulation, bio-distribution in the body and cellular internalization. Particles less than $10 \mathrm{~nm}$ in size are cleared easily from the body by kidney filtration, while larger ones have the tendency to be cleared by cells of the reticuloendothelial system (RES). Hence, nanoparticles with the optimal size should give favorable results. It was demonstrated that nanoparticles $100-200 \mathrm{~nm}$ size have the best properties for cellular uptake [25]. In order to synthesize monodispersed nanoparticles, a self-assembled polydispersed lipid suspension in water was further extruded thorough a $200 \mathrm{~nm}$ polycarbonate membrane (Step 4, Figure 3) repeatedly to get uniform particles with a size ranging from 100-200 nm. The nanoparticles synthesized from vitamin D3 were spherical in shape with a size less than $200 \mathrm{~nm}$. The size and morphology of nanoparticles were characterized by DLS (Figure 4), atomic force microscopy (AFM), field emission scanning electron microscopy (FESEM), and transmission electron microscopy (TEM) (Figure 5).

The drug loading was found to be very high when the vitamin drug conjugates were used in the nanoparticle formation compared to that of nanoparticles synthesized by the physical encapsulation of drugs.

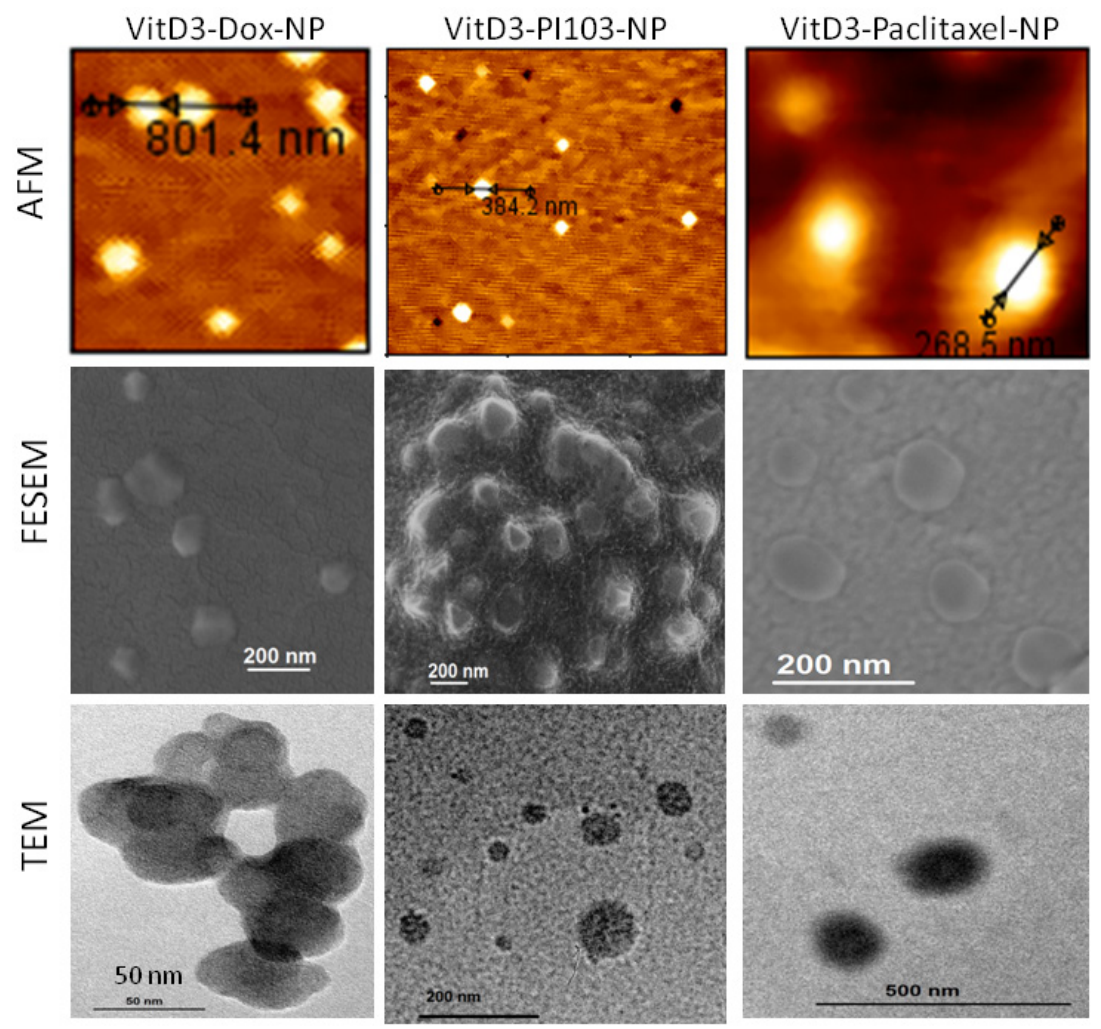

Figure 5. AFM, FESEM and TEM images of vitamin D3-drug loaded nanoparticles 
The loading of different drugs was quantified from a standard concentration versus absorbance graph in characteristic $\lambda_{\max }=340 \mathrm{~nm}, 480 \mathrm{~nm}$ and $270 \mathrm{~nm}$ for PI103, doxorubicin and paclitaxel, respectively, from ultraviolet-visible spectroscopy (UV-VIS). The loading efficiency of the drugs in the nanoformulation was calculated using the following equation.

$$
\text { Drug loading efficiency }=\frac{\text { Amount of drug present }(\mu \mathrm{g}) \text { in nanoparticle } \times 100 \%}{\begin{array}{c}
\text { Amount of vitamin D3-drug conjugate }(\mu \mathrm{g}) \\
\text { taken for nanoprticle synthesis }
\end{array}}
$$

The loading efficiency of drugs in vitD3-doxorubicin NPs, vitD3-paclitaxel NPs and vitD3-PI103 NPs was $21.7 \%, 36.8 \%$, and $26.9 \%$, respectively.

\subsubsection{Release of drugs from nanoparticles}

The release of drugs from nanoparticles is another important concern in drug delivery. Slow and sustained release of drugs is preferred over uncontrolled burst release, as burst release might lead to higher active drug concentrations near or above the toxic level in the blood circulation before reaching the tumor site [26]. Moreover, there is the possibility of active drug being metabolized and excreted without utilization, leading to wastage of drugs, both therapeutically and economically. When the drug is released slowly from nanoparticles over a period of time, this greatly reduces both the number and frequency of administrations necessary to maintain a therapeutic level of the drug in the body.

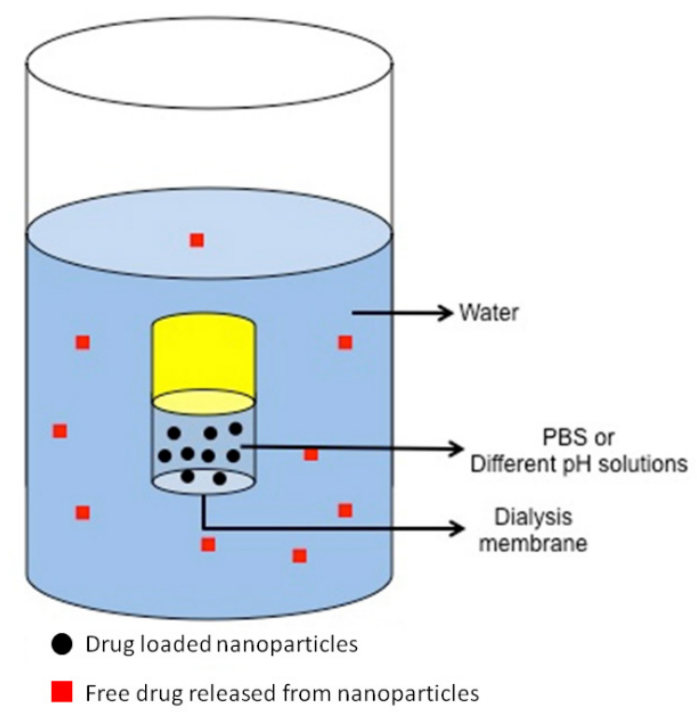

Figure 6. Schematic representation of drug release profile determination experiment by the dialysis method 
To observe the release profile of drugs from the nanoparticles, we incubated the nanoparticles at pH 5.5 (to mimic the acidic lysosomal compartments inside the cells) and at physiological pH 7.4 (to mimic the blood circulation environment) inside a dialysis membrane with molecular weight cut-off $(M W C 0)=1000$ Daltons (Figure 6). Aliquots of known amounts were taken at pre-determined time points from outside the dialysis bag and the release of the drugs was quantified by a concentration versus absorbance calibration graph from UV-VIS spectroscopy. From this release kinetics experiment, it was found that vitD3-paclitaxel NP released $75.56 \%$ of paclitaxel in $48 \mathrm{~h}$ at $\mathrm{pH} 5.5$, whereas only $34.88 \%$ paclitaxel was released at pH 7.4 in $130 \mathrm{~h}$. On the other hand, vitD3-PI103-NP released $77.74 \%$ of PI103 in $69 \mathrm{~h}$, whereas at pH 7.4 a similar amount, $74.03 \%$ of PI103, was released in 50 h. Finally, $66.35 \%$ of doxorubicin was released in $120 \mathrm{~h}$ at $\mathrm{pH} 5.5$ from the vitD3-Dox-NP, whereas at pH 7.4 only $9.47 \%$ of doxorubicin was released in $72 \mathrm{~h}$, which is seven-fold less release than at $\mathrm{pH}$ 5.5. From the release profile, it is clear that drugs were released in a slow, sustained manner over a period of time, which is expected to show better therapeutic efficacy when nanoparticles are injected intravenously into the body. It also showed enhanced release at $\mathrm{pH} 5.5$ compared to $\mathrm{pH} 7.4$; it was rationalized that the phenolic ester linkages, ester linkages and amide linkages in the vitD3-PI103 conjugate (3), vitD3-paclitaxel conjugate (4) and vitD3-doxorubicin conjugate (5), respectively, were more labile at $\mathrm{pH} 5.5$ compared to $\mathrm{pH} 7.4$, which resulted in enhanced release of PI103, paclitaxel and doxorubicin at an acidic $\mathrm{pH}$.

\subsubsection{In vitro cytotoxicity assay}

These drug-loaded vitamin D3 nanoparticles showed good cytotoxicity in an in vitro cell viability assay performed using the HeLa cervical cancer cell line. VitD3-paclitaxel-NPs showed cell death with $\mathrm{IC}_{50}=0.25 \mu \mathrm{M}$, inducing $17.78 \%$ cell viability, whereas free paclitaxel showed $\mathrm{IC}_{50}=0.092 \mu \mathrm{M}$ inducing $5.03 \%$ cell viability at $25 \mu \mathrm{M}$. VitD3-Dox-NPs showed cell death with $\mathrm{IC}_{50}=0.26 \mu \mathrm{M}$ compared to free doxorubicin with an $\mathrm{IC}_{50}=0.21 \mu \mathrm{M}$. VitD3-Dox-NPs induced $7.87 \%$ cell viability whereas free doxorubicin induced $1.37 \%$ cell viability at $25 \mu \mathrm{M}$. Finally, vitD3-PI103-NPs showed cell death with an $\mathrm{IC}_{50}=12.8 \mu \mathrm{M}$ inducing $38.65 \%$ cell viability, whereas free PI103 showed cell death with an $\mathrm{IC}_{50}=0.76 \mu \mathrm{M}$ inducing $5.38 \%$ cell viability at $25 \mu \mathrm{M}$ in $48 \mathrm{~h}$. 

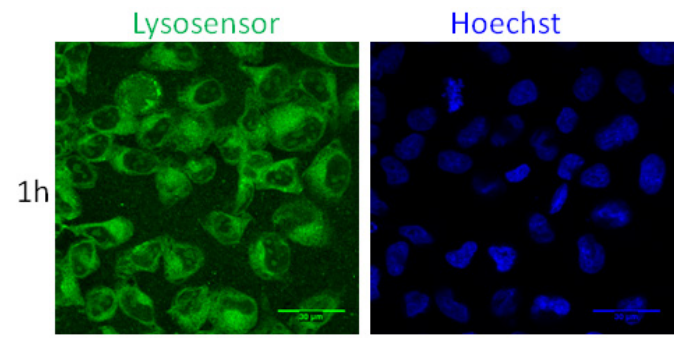

VitD3-Dox-NP
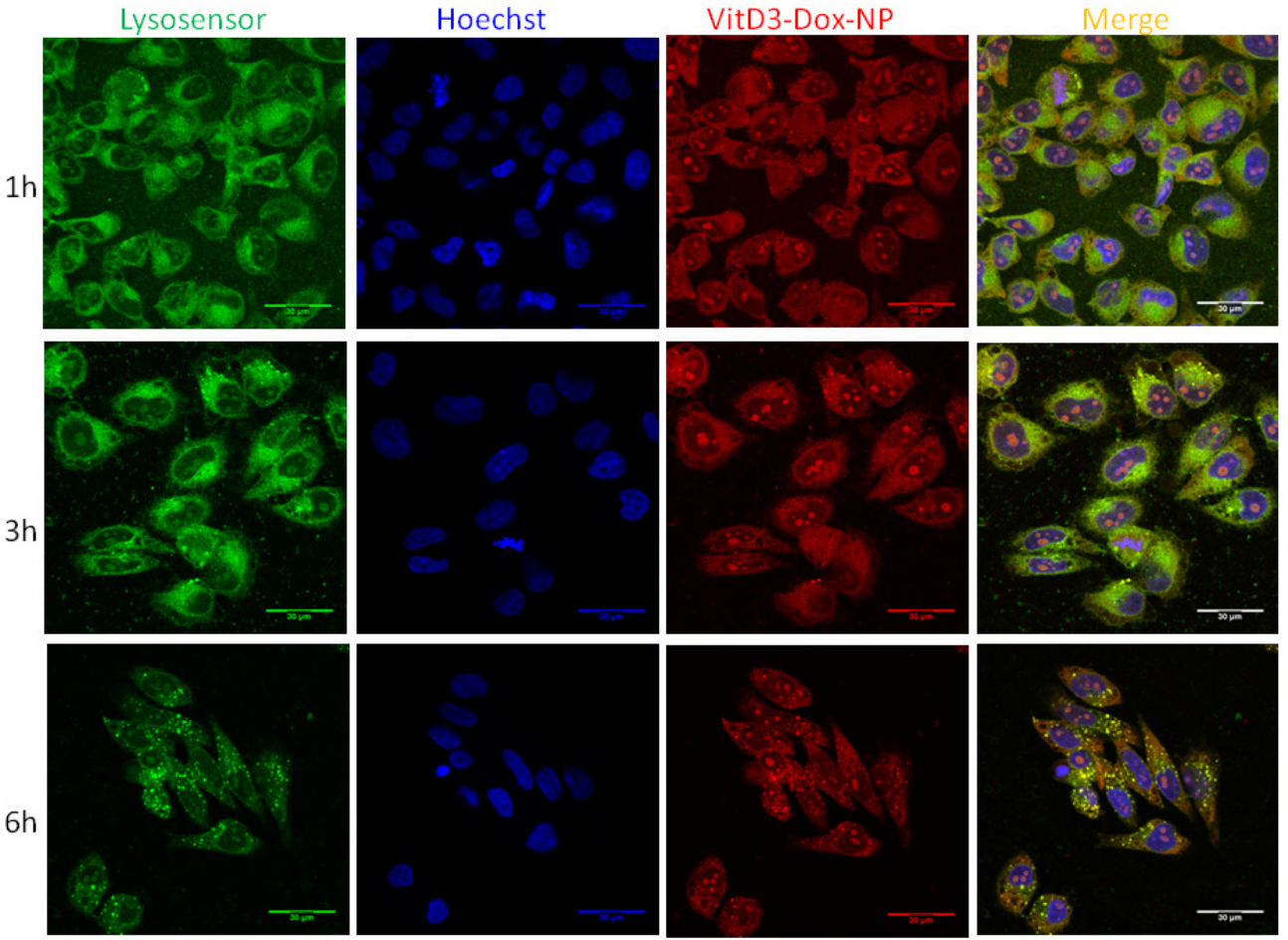

Figure 7. Internalization of vitD3-Dox-NP in HeLa cells in $1 \mathrm{~h}, 3 \mathrm{~h}$ and $6 \mathrm{~h}$ time points. Lysosomal compartments and nuclei were stained by LysoSensor (green) and Hoechst (blue) fluorescent dyes. The images were taken by confocal laser scanning microscopy (CLSM). Size bar $=30 \mu \mathrm{m}$.

It is known that nanoparticles enter into cells by different pathways such as phagocytosis, macropinocytosis, clathrin and caveolin mediated endocytosis, depending on their size, surface charge, etc. [27]. After internalization by different pathways, nanoparticles are trafficked to endosomes and then to sorting endosomes; from here, a fraction of nanoparticles is sorted back to the extracellular milieu through recycling endosomes while the remaining fraction is transported to secondary endosomes, followed by fusing with lysosomes where nanoparticles are expected to release their payloads [28]. To understand the cellular uptake mechanism of vitamin D3-NPs, we treated HeLa cells with vitD3-Dox-NPs and observed the internalization of NPs by CLSM; it was found that vitD3-Dox-NPs were internalized into HeLa cells through a low $\mathrm{pH}$ lysosomal compartment, whereas free doxorubicin internalized through the diffusion pathway (Figure 7). It can be anticipated that vitD3-paclitaxel-NPs and vitD3-PI103-NPs will also be internalized by a similar endocytosis mechanism through the low $\mathrm{pH}$ lysosomal compartment. 


\subsubsection{Dual drug loaded vitamin D3 nanoparticles}

The majority of successful cancer therapies are limited by the development of drug resistance [29]. Several cancers develop resistance to chemotherapy drugs by a mechanism called multidrug resistance (MDR), leading to the failure of many forms of chemotherapy [30]. One of several reasons for MDR is repeated treatment with single drug agents, resulting in resistance to chemotherapy. Moreover, since cancers are dependent on multiple altered molecular pathways, single agent therapies alone might not work to offer long-lasting benefits to patients [31]. Therefore, combination chemotherapy with two or more drugs is used to treat cancer cells to circumvent tumor drug resistance [32]. However, as discussed previously, current chemotherapy is limited by non-specific interactions, resulting in severe side effects which are even more severe when drug cocktails are administered. Also, it is very important to maintain controlled drug ratios to obtain optimal drug activity by combination therapy. However, this is difficult due to the different pharmacokinetic and pharmacodynamic profiles of drugs. Different types of polymeric nanoparticles [33-35], lipidic nanoparticles [36,37], iron oxide nanoparticles [38], nanocomplexes [39], and graphene oxide nanoparticles [40] have been used as delivery vehicles for the co-delivery of different anticancer drugs with unified pharmacokinetic profiles and controlled loading ratios.

To exploit these novel biocompatible vitamin D3 nanoparticles as a versatile platform, we also synthesized dual drug loaded nanoparticles by using vitamin D3-drug conjugates for the combinational drug delivery [41].

To obtain dual-drug loaded vitamin D3 nanoparticle, we mixed the vitD3-PI103 conjugate (3 in Figure 2) with PC and DSPE-PEG in a 1:2:0.2 weight ratio and the lipid film was formed in the same manner discussed in section 16.3.1. This drug loaded lipid film was further hydrated with a $1 \mathrm{mg} \mathrm{mL}^{-1}$ water solution of the second drug (doxorubicin or cisplatin or proflavine) to obtain vitD3-PI103-Dox-NP, vitD3-PI103-CDDP-NP and vitD3-PI103proflavine-NP, respectively.

These dual drug-loaded vitamin D3 nanoparticles also showed a considerable degree of improved loading. The mean drug loading in vitD3-PI103-CDDP-NP was found to be equal to $23.8 \mu \mathrm{g}$ of PI103 and $306.4 \pm 17.6 \mu \mathrm{g}$ of cisplatin (cisplatin encapsulation efficiency $=67.2 \%$ ) per $\mathrm{mg}$ of the formulation. Moreover, the mean drug loading in vitD3-PI103-Dox-NP was $42.0 \pm 2.5 \mu \mathrm{g}$ for PI103 and $158.0 \pm 7.4 \mu \mathrm{g}$ for doxorubicin (doxorubicin encapsulation efficiency $=34.2 \%$ ) per $\mathrm{mg}$ of the final formulation. Finally, the mean drug loading for PI103 and proflavine in vitD3-PI103-proflavine-NP were found to be equal to $66.4 \pm 9.4 \mu \mathrm{g}$ and $149.5 \pm 11.7 \mu \mathrm{g}$, respectively, (proflavine encapsulation efficiency $=32.2 \%$ ) per $\mathrm{mg}$ of the final formulation.

These nanoparticles also showed pH-triggered release, where nanoparticles were incubated at pH 5.5; vitD3-PI103-CDDP-NP showed a slow and sustained 
release profile for $72 \mathrm{~h}$, having released $79.22 \%$ of cisplatin in $23 \mathrm{~h}$ and $78.73 \%$ of PI103 in $47 \mathrm{~h}$. However, vitD3-PI103-proflavine-NP released $86.14 \%$ of proflavine in $48 \mathrm{~h}$ and $72.88 \%$ of PI103 in $96 \mathrm{~h}$. Finally, vitD3-PI103-Dox-NP demonstrated $47.05 \%$ release of doxorubicin in $72 \mathrm{~h}$ and $91.5 \%$ of PI103 release in $11 \mathrm{~h}$. At pH 7.4 and $37^{\circ} \mathrm{C}$, it was noticed that vitD3-PI103-CDDPNP released $41.5 \%$ of cisplatin in $47 \mathrm{~h}$ and $61.4 \%$ of PI103 in $25 \mathrm{~h}$. However, vitD3-PI103-Dox-NP showed $48.8 \%$ PI103 and $35.2 \%$ doxorubicin release in $72 \mathrm{~h}$. Finally, vitD3-PI103-proflavine-NP released $45 \%$ of proflavine in $96 \mathrm{~h}$ and $48 \%$ of PI103 in $72 \mathrm{~h}$.

Dual drug-loaded NPs also showed significant cytotoxicity in human hepatocellular carcinoma cells (Hep3B), and the cytotoxicity was considerably higher than single drug loaded nanoparticles, indicating synergistic effects. The vitD3-PI103-Dox-NP were cytotoxic to Hep3B cells with an $\mathrm{IC}_{50}=6.5 \mu \mathrm{M}$ compared to free doxorubicin showing an $\mathrm{IC}_{50}=18.4 \mu \mathrm{M}$. The vitD3-PI103-CDDP-NP showed a considerably lower $\mathrm{IC}_{50}=28.15 \mu \mathrm{M}$, or more cytotoxicity, compared to $\mathrm{IC}_{50}=49.08 \mu \mathrm{M}$ for free cisplatin. Finally, in contrast, vitD3-PI103-proflavine-NP showed a higher $\mathrm{IC}_{50}=30.5 \mu \mathrm{M}$ compared to $\mathrm{IC}_{50}=10.1 \mu \mathrm{M}$ for free proflavine. Whereas single drug encapsulated vitD3-Dox-NPs, vitD3-CDDP-NPs, and vitD3-Proflavine NPs showed an $\mathrm{IC}_{50}=87.7 \mu \mathrm{M}$ with $42.1 \%$ cell viability at $100 \mu \mathrm{M}, \mathrm{IC}_{50}=93.7 \mu \mathrm{M}$ with $44.5 \%$ cell viability at $100 \mu \mathrm{M}$ and $\mathrm{IC}_{50}=47.5 \mu \mathrm{M}$ with $12.6 \%$ cell viability at $80 \mu \mathrm{M}$, these cytotoxicities are considerably lower than the dual drug-loaded NPs.

Moreover, dual drug-loaded vitamin D3 nanoparticles showed enhanced cytotoxicity in a cisplatin resistant human hepatocellular carcinoma (Hep3B-R) cell line when compared to either monodrug loaded nanoparticles or the free single drug in a synergistic manner. VitD3-PI103-CDDP-NP showed a considerably lower $\mathrm{IC}_{50}=36.8 \mu \mathrm{M}$ with $24.0 \%$ viable cells compared to $\mathrm{IC}_{50}=70 \mu \mathrm{M}$ with $63.1 \%$ viable cells for free cisplatin in resistant cells, while vitD3-PI103-Dox-NP showed a considerably lower $\mathrm{IC}_{50}=9.66 \mu \mathrm{M}$ with $12.8 \%$ viable cells compared to $\mathrm{IC}_{50}=29.49 \mu \mathrm{M}$ with $13.9 \%$ viable cells for free doxorubicin. Finally, vitD3-PI103-proflavine-NP showed an $\mathrm{IC}_{50}=19.14 \mu \mathrm{M}$ with $8.7 \%$ viable cells compared to an $\mathrm{IC}_{50}=11.27 \mu \mathrm{M}$ for free proflavine with $2.5 \%$ viable cells. VitD3-Dox-NP, vitD3-CDDP-NP and vitD3-proflavine-NP induced $42.6 \%$ cell viability at $100 \mu \mathrm{M}$ with an $\mathrm{IC}_{50}=86.4 \mu \mathrm{M}, 40.3 \%$ cell viability at $100 \mu \mathrm{M}$ with an $\mathrm{IC}_{50}=79.3 \mu \mathrm{M}$, and $9.5 \%$ cell viability at $80 \mu \mathrm{M}$ with an $\mathrm{IC}_{50}=27.2 \mu \mathrm{M}$ respectively. Interestingly, vitD3-PI103-NP showed almost negligible cytotoxicity in Hep3B-R cells with an $\mathrm{IC}_{50}=92.9 \mu \mathrm{M}$. Finally, vitD3-PI103-CDDP-NP also showed considerable cytotoxicity in 5-fluorouracil (5-FU) resistant Hep3B cells (Hep3B-5FU-R) when the cell viability was evaluated at $24 \mathrm{~h}$ postincubation, showing an $\mathrm{IC}_{50}=37.9 \mu \mathrm{M}$ compared to $\mathrm{IC}_{50}=70 \mu \mathrm{M}$ for free $5-\mathrm{FU}$.

Dual drug-loaded nanoparticles showed anticancer activity in a synergistic way in both the Hep3B and Hep3B-R cell lines; this was calculated by the Chou-Talalay method [42]. In Hep3B cells, vitD3-PI103-CDDP-NP and vitD3-PI103- 
-Dox-NP showed combination index (CI) values that varied from 0.17-0.31, which clearly indicated the strong synergistic effect. However, vitD3-PI103-proflavine-NP showed slight synergy to a nearly additive effect with CI values varying from 0.62-1.02. Similarly, vitD3-PI103-CDDP-NP and vitD3-PI103-Dox-NP showed strong synergy ( $\mathrm{CI}=0.14-5.0)$ in Hep3B-R cells in contrast to vitD3-PI103-proflavine-NP, which showed weak synergy to a nearly additive effect (CI $=0.60-0.91)$.

These dual drug loaded vitamin D3 nanoparticles induced cytotoxicity by DNA damage, which was confirmed by western blot analysis with the quantification of poly(ADP-ribose) polymerase (PARP) as a marker for DNA damage repair [43]. For vitD3-PI103-CDDP-NP and vitD3-PI103-Dox-NP, greater cleaved PARP expression was observed (the carboxy-terminal catalytic domain has a molecular weight of $89 \mathrm{kDa}$ ) compared to that without treatment and with 5-fluorouracil (5-FU, a positive control) treatment in Hep3B cells.

Hence, in conclusion, we have developed a biodegradable-biocompatible novel vitamin D3 nanoparticle with a size less than $200 \mathrm{~nm}$, ideal for tumor homing through the EPR effect. This vitamin D3 nanoparticle can be used as platform to load single or multiple drugs with high loading. Drugs are released in a slow and sustained manner over a long period of time in a $\mathrm{pH}$ dependent manner. Furthermore, these nanoparticles were internalized into the tumor cells via endocytosis into the acidic lysosomal compartment and showed efficacy in cervical cancer cells as well as drug resistant hepatocellular carcinoma. We anticipate that these vitamin D3 nanoparticles can be translated to the clinic as a biocompatible and biodegradable vector for delivering drug combinations to reduce the toxic side effects of drug cocktails and to offer a better quality of life to cancer patients. 


\section{REFERENCES}

1. World cancer factsheet January 2014 - Cancer Research UK, http://publications.cancerresearchuk.org/downloads/product/CS_REPORT_ WORLD.pdf. (20/02/2015)

2. $\quad$ S.K. Carter, M.Slavik. Annu. Rev. Pharmacol. 14 (1974) 157-183.

3. D. Kakde, D. Jain, V. Shrivastava, R. Kakde, A. Patil. J. Appl. Pharmaceut. Sci. 1 (2011) 1-10.

4. $\quad$ R. Goyal, S. Kumar. J. Innov. Biol. 1 (2014) 84-96.

5. A. Kumar, F. Chen, A. Mozhi, X. Zhang, Y. Zhao, X. Xue, Y. Hao, X. Zhang, P.C. Wang, X.-J. Liang. Nanoscale 5 (2013) 8307-8325.

6. D.F. Emerich, C.G. Thanos. J. Drug Target. 15 (2007) 163-183.

7. I. Brigger, C. Dubernet, P. Couvreur. Adv. Drug Deliv. Rev. 54 (2002) 631-651.

8. J.N. Weinstein, L.D. Leserman. Pharmacol. Ther. 24 (1984) 207-233.

9. A.D. Bangham. Bioessays 17 (1995) 1081-1088.

10. D. Bhadra, S. Bhadra, S. Jain, N.K. Jain. Intl. J. Pharm. 257 (2003) 111-124.

11. Y. Cheng, Z. Xu, M. Ma, T. Xu. J. Pharmaceut. Sci. 97 (2008) 123-143.

12. K. Kataoka, A. Harada, Y. Nagasaki. Adv. Drug Deliv. Rev. 47 (2001) 113-131.

13. N. Nasongkla, E. Bey, J. Ren, H. Ai, C. Khemtong, J.S. Guthi, S.-F. Chin, A.D. Sherry, D.A. Boothman, J. Gao. Nano Lett. 6 (2006) 2427-2430.

14. F. Ahmed, R.I. Pakunlu, A. Brannan, F. Bates, T. Minko, D.E. Discher. J. Control. Rel. 116 (2006) 150-158.

15. R.H. Müller, K. Mader, S. Gohla. Eur. J. Pharm. Biopharm. 50 (2000) 161-177.

16. G. Han, P. Ghosh, V.M. Rotello. Nanomedicine 2 (2007) 113-123.

17. I.I. Slowing, J.L. Vivero-Escoto, C.-W. Wu, V.S.-Y. Lin. Adv. Drug Deliv. Rev. 60 (2008) 1278-1288.

18. R. Savla, O. Taratula, O. Garbuzenko, T. Minko. J. Control. Rel. 153 (2011) 16-22.

19. Z. Liu, K. Chen, C. Davis, S. Sherlock, Q. Cao, X. Chen, H. Dai. Cancer Res. 68 (2008) 6652-6660.

20. M. Palombo, M. Deshmukh, D. Myers, J. Gao, Z. Szekely, P.J. Sinko. Annu. Rev. Pharmacol. Toxicol. 54 (2014) 581-598.

21. T.M. Allen, P.R. Cullis. Science 303 (2004) 1818-1822.

22. D. Peer, J.M. Karp, S. Hong, O.C. Farokhzad, R. Margalit, R. Langer. Nat. Nanotechnol. 2 (2007) 751-760.

23. S. Patil, S. Gawali, S. Patil, S. Basu. J. Mater. Chem. B 1 (2013) 5742-5750.

24. H.H. Glossmann. J. Invest. Dermatol. 130 (2010) 2139-2141.

25. $\quad$ R.A. Petros, J.M. DeSimone. Nat. Rev. Drug Discov. 9 (2010) 615-627.

26. X. Huang, C.S. Brazel. J. Control. Rel. 73 (2001) 121-136.

27. T.-G. Iversen, T. Skotland, K. Sandvig. Nano Today 6 (2011) 176-185.

28. L. Rajendran, H.-J. Knolker, K. Simons. Nat. Rev. Drug Discov. 9 (2010) 29-42.

29. L.A. Garraway, P.A. Jänne. Cancer Discov. 2 (2012) 214-226.

30. A. Persidis. Nat. Biotechnol. 17 (1999) 94-95.

31. R.W. Humphrey, L.M. Brockway-Lunardi, D.T. Bonk, K.M. Dohoney, J.H. Doroshow, S.J. Meech, M.J. Ratain, S.L. Topalian, D.M. Pardoll. J. Natl. Cancer Inst. 103 (2011) 1222-1226.

32. P. Parhi, C. Mohanty, S.K. Sahoo. Drug Discov. Today 17 (2012) 1044-1052.

33. N. Kolishetti, S. Dhar, P.M. Valencia, L.Q. Lin, R. Karnik, S.J. Lippard, R. Langer, O.C. Farokhzad. Proc. Natl. Acad. Sci. U. S. A. 107 (2010) 17939-17944. 
34. L.E. van Vlerken, Z. Duan, M.V. Seiden, M.M. Amiji. Cancer Res. 67 (2007) 4843-4850.

35. L. Liao, J. Liu, E.C. Dreaden, S.W. Morton, K.E. Shopsowitz, P.T. Hammond, J.A. Johnson. J. Am. Chem. Soc. 136 (2014) 5896-5899.

36. R.J. Lee. Mol. Cancer Ther. 5 (2006) 1639-1640.

37. D. Cosco, D. Paolino, F. Cilurzo, F. Casale, M. Fresta. Int. J. Pharm. 422 (2012) 229-237.

38. F. Dilnawaz, A. Singh, C. Mohanty, S.K. Sahoo. Biomaterials 31 (2010) 3694-3706.

39. C. Wang, H. Xu, C. Liang, Y. Liu, Z. Li, G. Yang, L. Cheng, Y. Li, Z. Liu. ACS Nano 7 (2013) 6782-6795.

40. L. Zhang, J. Xia, Q. Zhao, L. Liu, Z. Zhang. Small 6 (2010) 537-544.

41. S. Palvai, J. Nagraj, N. Mapara, R. Chowdhury, S. Basu. RSC Adv. 4 (2014) 57271-57281.

42. T.-C. Chou. Cancer Res. 70 (2010) 440-446.

43. P. Bouwman, J. Jonkers. Nat. Rev. Cancer 12 (2012) 587-598. 